\title{
Hyperchloremia is associated with 30-day mortality in major trauma patients: a retrospective observational study
}

Jin Young Lee ${ }^{1,2}$, Tae Hwa Hong ${ }^{1,2}$, Kyung Won Lee ${ }^{1,2}$, Myung Jae Jung ${ }^{1,2}$, Jae Gil Lee ${ }^{1,2}$ and Seung Hwan Lee ${ }^{1,2^{*}}$ (D)

\begin{abstract}
Background: Chloride is important for maintaining acid-base balance, muscular activity, osmosis and immunomodulation. In patients with major trauma, chloride levels increase after fluid therapy; this is associated with poor clinical outcomes. The purpose of this study was to determine whether hyperchloremia was associated with increased mortality in patients who had sustained major trauma.

Methods: This study enrolled 266 major trauma patients by retrospective chart review, from January 2011 to December 2015. Patients were older than 16 years; were admitted to an intensive care unit; survived more than 48 h; and had sustained major trauma, defined as an injury severity score $\geq 16$. Hyperchloremia was defined as a chloride level $>110 \mathrm{mEg} / \mathrm{L}$. Delta chloride ( $\Delta$ chloride) was defined as the difference between the serum chloride level measured 48-h post-admission and the initial level. Clinical and laboratory variables were compared between survivors $(n=235)$ and non-survivors $(n=31)$. A multivariate logistic regression analysis was performed to assess the association between hyperchloremia 48-h post-admission (hyperchloremia-48) and 30-day mortality.
\end{abstract}

Results: The overall 30-day mortality was $11.7 \%(n=31)$. Hyperchloremia-48 occurred in 65 patients $(24.4 \%)$ and the incidence was significantly different between survivors and non-survivors (19.6 vs. $61.3 \%$, respectively, $p<0.001$ ). Multivariate logistic analysis identified hyperchloremia-48 and $\Delta$ chloride as independent predictive factors for 30 -day mortality in major trauma patients.

Discussion: Infusion of chloride-rich solutions, such as normal saline, is itself associated with hyperchloremia, which has been associated with poor patient outcomes. Patients receiving normal saline were more likely to suffer major postoperative complications, acute kidney injury, and infections. Moreover, large changes in serum chloride levels correlated with greater in-hospital mortality.

Conclusion: Hyperchloremia 48-h post-admission and $\Delta$ chloride was associated with 30-day mortality in major trauma patients. These indices may be useful prognostic markers.

Keywords: Chloride, Mortality, Trauma, Normal saline, Resuscitation

\footnotetext{
* Correspondence: seunghwan@yuhs.ac

This abstract was presented at the 17th European Congress of Trauma \& Emergency surgery, held on April 24-26, 2016, in Vienna, Austria.

'Division of Critical Care and Trauma Surgery, Department of Surgery, Yonsei University College of Medicine, 50-1 Yonsei-ro, Seodaemun-gu, Seoul 03722, Republic of Korea

${ }^{2}$ Trauma Training Center, Severance Hospital, Yonsei University Health

System, Seoul, Republic of Korea
} 


\section{Background}

Chloride is the major anion in blood, accounting for approximately one-third of plasma tonicity, for $97-98 \%$ of all strong anionic charges, and for two-thirds of all negative charges in plasma [1]. It plays a pivotal role in many body functions, including acid-base balance, muscular activity, osmosis and immunomodulation [2]. Despite its physiologic importance, chloride has received much less attention than other routinely measured electrolytes [1]. However, the more our understanding of acid-base and chloride channel physiology increases, the greater our interest in chloride becomes. In human studies, hyperchloremia has been identified as being able to reduce splanchnic blood flow, as measured by gastric tonometry [3]. Renal vasoconstriction has been induced experimentally by infusion of chloride-containing solutions in animal studies $[4,5]$. In experimental sepsis, hyperchloremic acidosis provoked an increase in circulating inflammatory molecules [6]. Previous studies have reported an association between hyperchloremia and mortality in critically ill patients $[7,8]$, and in patients following non-cardiac surgery [9].

Severely injured trauma patients often initially require significant fluid resuscitation. According to Advanced Trauma Life Support guidelines, a bolus of 1-2 L of a warmed isotonic solution may be required to achieve an appropriate response in hemodynamically unstable patients [10]. The fluids most commonly used for resuscitation are $0.9 \%$ normal saline (NS, $0.9 \% \mathrm{NaCl}$ ), which contains supra-physiologic levels of sodium and chloride (154 mmol/L), and "balanced" crystalloids, such as Ringer's lactate or Plasma-Lyte (Baxter Healthcare, Deerfield, IL, USA), which typically have more physiologic electrolyte concentrations (98-112 mmol/L chloride and 130-140 mmol/L sodium) [11]. Because it contains supra-physiologic levels of sodium and chloride, NS can cause hyperchloremic metabolic acidosis $[12,13]$. As major trauma patients are commonly exposed to NS during the salvage phase of shock, they are susceptible to hyperchloremia in the post-resuscitation phase. The purpose of this study was to determine whether serum chloride levels are associated with mortality in patients who have sustained major trauma.

\section{Methods}

\section{Study design and data collection}

We conducted a retrospective observational study in single center from January 2011 to December 2015. A total of 1095 trauma patients were admitted to the emergency department during this period. We excluded patients with the following characteristics: $\leq 16$ years of age; injury severity score (ISS) < 16; admitted to a general ward; underlying chronic renal failure; died within $48 \mathrm{~h}$ of admission to the emergency department; and with initial hyperchloremia (chloride $>110 \mathrm{mmol} / \mathrm{L}$ ). The patients with initial hyperchloremia $(n=18)$ had been transferred from other hospitals after receiving fluid resuscitation or other management. They were also transferred 3 days or more after injury. Consequently, 829 patients were excluded and 266 enrolled. Patients were divided into two groups: survivors $(n=235)$ and non-survivors $(n=31)$. These groups were compared with respect to clinical and biochemical variables (Fig. 1).

The study was approved by the Institutional Review Board (IRB No. 4-2016-0295), which waived the requirement for informed consent because of the retrospective nature of the study.

\section{Study variables and definition}

Baseline characteristics included age, sex, underlying disease, Glasgow coma scale (GCS) score, and trauma related variables, such as ISS, revised trauma score (RTS), trauma and injury severity score (TRISS), mechanism(s) of injury and injury regions. Laboratory results were measured at initial presentation to the emergency unit at 48-h post-admission. Data regarding the length of hospital stay, length of intensive care unit stay and duration of mechanical ventilation were collected and analyzed.

Hyperchloremia was defined as a serum chloride level $>110 \mathrm{mmol} / \mathrm{L}$. Delta chloride $(\Delta$ chloride) was defined as the difference between the chloride level 48-h post-admission and the initial level. Body mass index was defined as body mass divided by the square of height $\left(\mathrm{kg} / \mathrm{m}^{2}\right)$, and GCS score refers to that obtained at the initial assessment. The Acute Physiology and Chronic Health Evaluation II (APACHE II) scores were calculated within the first day of ICU admission. Hypotension was defined as an initial systolic blood pressure $<90 \mathrm{mmHg}$, and hypothermia was defined as an initial body temperature $<35{ }^{\circ} \mathrm{C}$. Emergency operations and emergency angio-embolization were defined as those performed within $24 \mathrm{~h}$ of admission. The total amount of fluid administered over $48 \mathrm{~h}$ was included, not only crystalloids but also colloids and blood transfusion products. NS infused over the first $48 \mathrm{~h}$ included fluid for resuscitation as well as that mixed with other drugs. Acute kidney injury (AKI) was defined according to the consensus Risk, Injury, Failure, Loss and EndStage Renal Disease (RIFLE) definition [14] using serum creatinine changes with urine output criteria.

\section{Study outcomes}

The primary outcome investigated in the multivariate logistic regression was 30-day hospital mortality, defined as all-cause mortality within 30-days of hospital admission. 


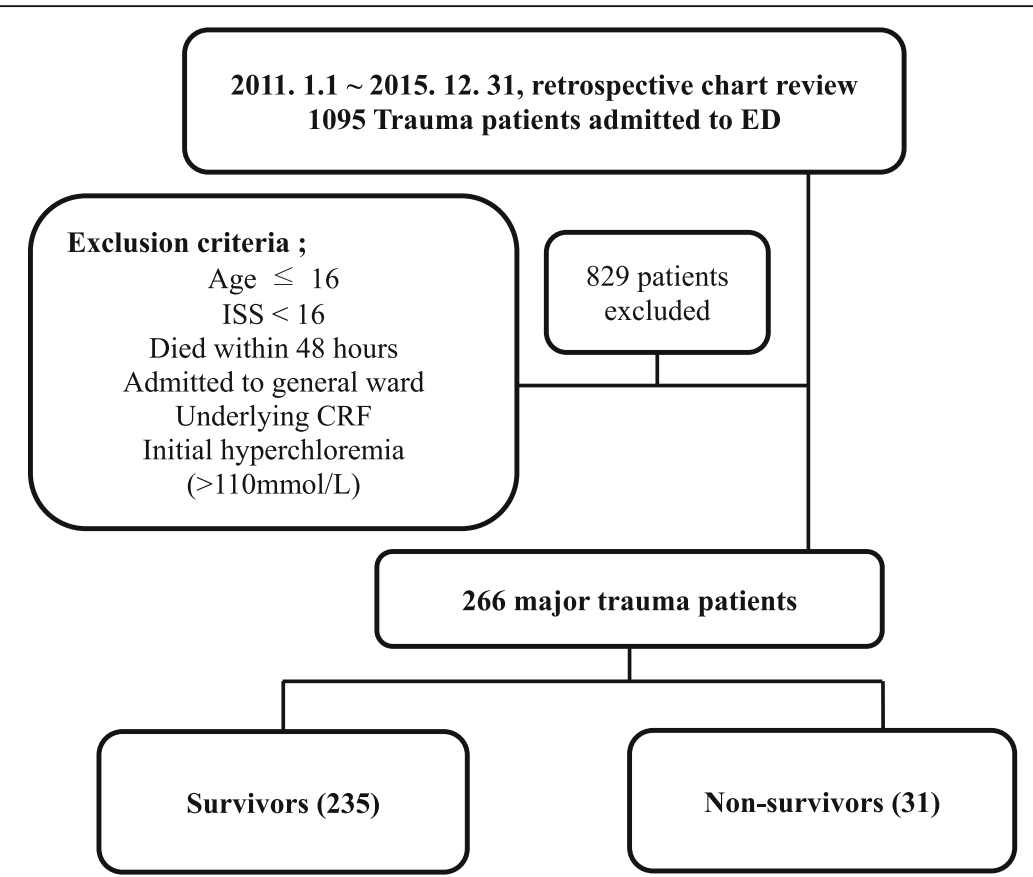

Fig. 1 Study population by inclusion and exclusion criteria. ED, emergency department; ISS, injury severity score; CRF, chronic renal failure

\section{Statistical analysis}

Statistical analysis was performed using IBM SPSS Statistics 20.0 (IBM Co. Armonk, NY). Categorical data were presented as number (\%), and were compared using the chi-square or Fisher's exact test. Continuous variables were expressed as the mean and standard deviation or median and inter-quartile range (IQR), and were compared between groups using the Student's $t$-test or Mann-Whiney $U$ test. Factors found to be significantly associated with mortality $(p$-value $<0.05)$ by univariate analysis were included in the multivariate analysis with age, as age is regarded as an important factor for mortality. APACHE II score was excluded from the analysis, because most of the variables that comprise the APACHE II score were included individually. Laboratory factors used in the analysis, such as hyperchloremia and base deficit, were the results at 48-h post-admission. Logistic regression analysis was performed independently to identify the association of 30-day mortality with hyperchloremia-48, absolute value of chloride and $\Delta$ chloride by using the maximum likelihood method and backward stepwise selection. Goodness-of-fit was assessed using the Hosmer-Lemeshow test.

\section{Results}

Of the 266 patients included in the study, 235 (88 \%) survived and 31 (12\%) died within 30 days of admission. The mean age of the patients was 49.7 years and 196 (74\%) patients were male with no significant difference between survivors and non-survivors in terms of age and sex. Mean ISS, RTS and APACHE II scores differed significantly between the two groups (Table 1).

Hypotension and hypothermia were detected in 22 (71 $\%)$ and 10 (32\%) non-survivors, respectively. Both these proportions were significantly greater compared to those of survivors (Table 2). There was a significant difference between survivors and non-survivors in terms of receiving blood-product transfusions (50\% vs. $87 \%$, respectively, $p<0.001)$. The median volume of fluid infused during the first $48 \mathrm{~h}$ was also significantly different between survivors and non-survivors (7.4 L vs. $11.8 \mathrm{~L}, p<0.001)$, but volumes of NS infused were not significantly different (2.0 L vs. $2.1 \mathrm{~L}, p=0.75$ ). Overall, the median positive fluid balance was $2.2 \mathrm{~L}$, significantly higher in non-survivors than in survivors $(2.1 \mathrm{~L}$ vs. $2.7 \mathrm{~L}$ cumulative fluid balance at $48 \mathrm{~h}, p=0.03)$.

There were no significant differences in electrolyte levels at initial measurement. However, $48 \mathrm{~h}$ postadmission, sodium $(140.6 \pm 4.31 \mathrm{mmol} / \mathrm{L}$ vs. $148.5 \pm 10.1$ $\mathrm{mmol} / \mathrm{L}, p<0.001)$ and chloride $(105.7 \pm 4.7 \mathrm{mmol} / \mathrm{L}$ vs $112.6 \pm 10.7 \mathrm{mmol} / \mathrm{L}, p=0.002$ ) levels were significantly higher in non-survivors than in survivors, respectively. Base deficit and lactate levels also differed significantly between the two groups. The difference in chloride level between the 48-h post-admission and initial measurement, $\Delta$ chloride, was significantly higher in non-survivors $(10.3 \pm 11.1 \mathrm{mmol} / \mathrm{L})$ than in survivors $(1.7 \pm 5.2 \mathrm{mmol} / \mathrm{L}$, $p<0.001)$. In addition, increases in $\Delta$ chloride were significantly different between the two groups (Table 3 ). 
Table 1 Baseline characteristics of major trauma patients

\begin{tabular}{|c|c|c|c|c|}
\hline & Total (266) & Survivors (235) & Non-survivors (31) & $p$ value \\
\hline Age (years), mean $\pm S D$ & $49.7 \pm 19.5$ & $49.1 \pm 19.7$ & $54.2 \pm 17.6$ & 0.17 \\
\hline Male, $n(\%)$ & $196(74)$ & $173(74)$ & $23(74)$ & 1.00 \\
\hline \multicolumn{5}{|l|}{ Underlying disease, $n$ (\%) } \\
\hline HTN & $50(19)$ & $42(18)$ & $8(26)$ & 0.33 \\
\hline DM & $32(12)$ & $27(12)$ & $5(16)$ & 0.55 \\
\hline Pulmonary tuberculosis & $4(2)$ & $3(1)$ & $1(3)$ & 0.39 \\
\hline Hepatitis & $3(1)$ & $2(1)$ & $1(3)$ & 0.31 \\
\hline $\mathrm{BMI}$, mean $\pm \mathrm{SD}$ & $23.2 \pm 3.0$ & $23.2 \pm 3.0$ & $23.7 \pm 2.9$ & 0.36 \\
\hline GCS, median (IQR) & $15.0(7.0-15.0)$ & $15.0(9.0-15.0)$ & $5.0(3.0-12.0)$ & $<0.001$ \\
\hline ISS, mean \pm SD & $26.0 \pm 7.9$ & $25.4 \pm 7.6$ & $30.8 \pm 8.4$ & $<0.001$ \\
\hline RTS, mean $\pm S D$ & $6.726 \pm 1.684$ & $6.939 \pm 1.465$ & $5.112 \pm 2.298$ & $<0.001$ \\
\hline TRISS, mean \pm SD & $79.1 \pm 26.8$ & $83.7 \pm 23.0$ & $47.0 \pm 32.2$ & $<0.001$ \\
\hline APACHE II, mean \pm SD & $16.2 \pm 9.2$ & $14.7 \pm 8.3$ & $27.1 \pm 8.5$ & $<0.001$ \\
\hline Injury mechanism, n (\%) & & & & 0.26 \\
\hline MVA (pedestrian) & $86(32)$ & $73(31)$ & $13(42)$ & \\
\hline MVA (passenger) & $39(15)$ & $38(16)$ & $1(3)$ & \\
\hline Motorcycle accidents & $58(22)$ & $53(23)$ & $5(16)$ & \\
\hline Falls & $63(24)$ & $53(23)$ & $10(32)$ & \\
\hline Penetrating injuries & $5(2)$ & $5(2)$ & $0(0)$ & \\
\hline Others & $15(6)$ & $13(6)$ & $2(7)$ & \\
\hline \multicolumn{5}{|l|}{ Injury region, $n$ (\%) } \\
\hline Head \& Neck & $209(79)$ & $182(77)$ & $27(87)$ & 0.25 \\
\hline Face & $105(40)$ & $94(40)$ & $11(36)$ & 0.70 \\
\hline Chest & $188(71)$ & $172(73)$ & $16(52)$ & 0.02 \\
\hline Abdomen & $143(54)$ & $127(54)$ & $16(52)$ & 0.85 \\
\hline Extremities & $179(67)$ & $160(68)$ & $19(61)$ & 0.54 \\
\hline External & $193(73)$ & $171(73)$ & $22(71)$ & 1.00 \\
\hline
\end{tabular}

SD standard deviation, DM diabetes mellitus, BMI body mass index, GCS Glasgow coma scale, ISS injury severity score, RTS revised trauma score, TRISS, trauma and injury severity score, APACHE II acute physiology and chronic health evaluation II, MVA motor vehicle accidents

Table 2 Comparison of clinical parameters between survivors and non-survivors

\begin{tabular}{|c|c|c|c|c|}
\hline & Total (266) & Survivors (235) & Non-survivors (31) & $p$ value \\
\hline Hypotension (SBP < 90), n (\%) & $105(40)$ & $83(35)$ & $22(71)$ & $<0.001$ \\
\hline 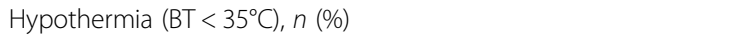 & $36(13)$ & $26(11)$ & $10(32)$ & 0.003 \\
\hline \multicolumn{5}{|l|}{ Drug exposure during 48 h, $n$ (\%) } \\
\hline Vasopressor & $107(40)$ & $78(33)$ & $29(94)$ & $<0.001$ \\
\hline Loop diuretics & $123(46)$ & $101(43)$ & $22(71)$ & 0.004 \\
\hline NSAIDs & $46(17)$ & $43(18)$ & $3(10)$ & 0.32 \\
\hline Aminoglycoside & $27(10)$ & $25(11)$ & $2(7)$ & 0.55 \\
\hline Transfusion, $n(\%)$ & $144(54)$ & $117(50)$ & $27(87)$ & $<0.001$ \\
\hline Total volume of infused fluid during $48 \mathrm{~h} \mathrm{(L)}$, median (IQR) & $7.6(6.3-10.1)$ & $7.4(6.2-9.3)$ & $11.8(8.2-14.6)$ & $<0.001$ \\
\hline Total volume of infused NS during $48 \mathrm{~h}(\mathrm{~L})$, median (IQR) & $2.0(1.2-3.2)$ & $2.0(1.2-3.2)$ & $2.1(1.3-3.2)$ & 0.75 \\
\hline Total volume of infused Plasmalyte during $48 \mathrm{~h}(\mathrm{~L})$, median (IQR) & $2.0(1.0-3.0)$ & $2.0(1.0-3.0)$ & $3.0(1.0-3.6)$ & 0.22 \\
\hline Cumulative fluid balance at $48 \mathrm{~h}(\mathrm{~L})$, median (IQR) & $2.2(1.2-4.0)$ & $2.1(1.1-3.7)$ & $2.7(1.8-6.8)$ & 0.03 \\
\hline
\end{tabular}


Table 3 Comparison of laboratory findings between survivors and non-survivors

\begin{tabular}{|c|c|c|c|c|}
\hline & Total (266) & Survivors (235) & Non-survivors (31) & $p$ value \\
\hline \multicolumn{5}{|l|}{ Creatinine $(\mu \mathrm{mol} / \mathrm{L})$, mean $\pm S D$} \\
\hline Initial & $79.6 \pm 38.9$ & $79.6 \pm 39.8$ & $88.4 \pm 25.6$ & 0.20 \\
\hline At $48 \mathrm{~h}$. & $79.6 \pm 21.2$ & $79.6 \pm 39.8$ & $132.6 \pm 98.1$ & 0.001 \\
\hline \multicolumn{5}{|l|}{ Sodium $(\mathrm{mmol} / \mathrm{L})$, mean $\pm \mathrm{SD}$} \\
\hline Initial & $140.6 \pm 2.9$ & $140.6 \pm 2.7$ & $140.6 \pm 3.5$ & 0.93 \\
\hline At $48 \mathrm{~h}$ & $141.5 \pm 5.8$ & $140.6 \pm 4.3$ & $148.5 \pm 10.1$ & $<0.001$ \\
\hline \multicolumn{5}{|l|}{ Potassium (mmol/L), mean \pm SD } \\
\hline Initial & $3.9 \pm 0.5$ & $3.9 \pm 0.5$ & $4.0 \pm 0.6$ & 0.63 \\
\hline At $48 \mathrm{~h}$ & $3.8 \pm 0.5$ & $3.8 \pm 0.4$ & $3.8 \pm 0.8$ & 0.84 \\
\hline \multicolumn{5}{|l|}{ Chloride $(\mathrm{mmol} / \mathrm{L})$, mean $\pm \mathrm{SD}$} \\
\hline Initial & $103.9 \pm 3.3$ & $104.1 \pm 3.2$ & $102.3 \pm 3.9$ & 0.006 \\
\hline At $48 \mathrm{~h}$ & $106.5 \pm 6.1$ & $105.7 \pm 4.7$ & $112.6 \pm 10.7$ & 0.002 \\
\hline$\Delta$ chloride $(\mathrm{mmol} / \mathrm{L})$, mean $\pm \mathrm{SD}$ & $2.6 \pm 6.6$ & $1.7 \pm 5.2$ & $10.3 \pm 11.1$ & $<0.001$ \\
\hline$\Delta$ chloride $(\mathrm{mmol} / \mathrm{L}), n(\%)$ & & & & $<0.001$ \\
\hline$-10 \leq<0$ & $80(32)$ & $77(34)$ & $3(11)$ & \\
\hline $0 \leq<10$ & $148(59)$ & $134(60)$ & $14(52)$ & \\
\hline $10 \leq<20$ & $18(7)$ & $12(5)$ & $6(2)$ & \\
\hline $20 \leq<30$ & $5(2)$ & $2(1)$ & $3(11)$ & \\
\hline $40 \leq$ & $1(1)$ & $0(0)$ & $1(4)$ & \\
\hline \multicolumn{5}{|l|}{$\mathrm{pH}$, mean $\pm \mathrm{SD}$} \\
\hline Initial & $7.36 \pm 0.10$ & $7.37 \pm 0.88$ & $7.32 \pm 0.18$ & 0.16 \\
\hline At $48 \mathrm{~h}$ & $7.50 \pm 1.33$ & $7.52 \pm 1.43$ & $7.37 \pm 0.09$ & 0.59 \\
\hline \multicolumn{5}{|l|}{ Base deficit (mmol/L), mean \pm SD } \\
\hline Initial & $-5.7 \pm 4.0$ & $-5.4 \pm 3.8$ & $-8.2 \pm 4.6$ & 0.003 \\
\hline At $48 \mathrm{~h}$ & $-1.0 \pm 3.0$ & $-0.6 \pm 2.7$ & $-3.4 \pm 4.1$ & $<0.001$ \\
\hline \multicolumn{5}{|l|}{ Lactate $(\mathrm{mmol} / \mathrm{L})$, mean $\pm \mathrm{SD}$} \\
\hline Initial & $3.9 \pm 3.1$ & $3.6 \pm 2.7$ & $6.4 \pm 4.4$ & 0.003 \\
\hline At $48 \mathrm{~h}$ & $1.8 \pm 1.6$ & $1.5 \pm 1.3$ & $3.4 \pm 2.9$ & 0.03 \\
\hline
\end{tabular}

The median overall length of hospital stay was longer for survivors (25.0 days) than non-survivors (8.0 days, $p<0.001$ ), although there was no difference in the median duration of ICU stay. While emergency operations were more frequently performed in non-survivors, there was no difference between the groups in the proportion of patients undergoing angio-embolization. Hyperchloremia 48-h post-admission (hyperchloremia-48) was more common in non-survivors (Table 4).

Multivariate analysis revealed that hyperchloremia- 48 (adjusted odds ratio $[\mathrm{OR}]=4.567,95 \%$ confidence interval $[\mathrm{CI}]=1.634-12.764, p=0.004)$, RTS, base deficit, use of vasopressor and AKI were independent predictors for 30-day mortality in major trauma patients (Table 5). In addition, the absolute value of chloride at $48 \mathrm{~h}(\mathrm{OR}=$ $1.075,95 \% \mathrm{CI}=1.006-1.150, p=0.03$, Table 6) and $\Delta$ chloride $(\mathrm{OR}=1.096,95 \% \mathrm{CI}=1.027-1.169, p=0.006$,
Table 7) were independent risk factors for 30-day mortality in major trauma patients.

\section{Discussion}

This study comprised seriously injured patients (ISS $\geq$ 16) admitted to a trauma ICU. Hyperchloremia occurred frequently in patients who had normal levels of serum chloride on admission; $24 \%$ had serum chloride levels > $110 \mathrm{mmol} / \mathrm{L}$ 48-h post-admission. Multivariate logistic regression analysis identified hyperchloremia- 48 and $\Delta$ chloride as being independently associated with 30 -day mortality, adjusted for other significant variables. Similar to previous studies that reported an association between hyperchloremia and mortality in critically ill $[7,8]$, and postoperative patients [9], our study showed that elevated chloride levels may be associated with mortality in major trauma patients. 
Table 4 Clinical outcomes of survivors and non-survivors

\begin{tabular}{|c|c|c|c|c|}
\hline & Total (266) & Survivors (235) & Non-survivors (31) & $p$ value \\
\hline LoH (day), median (IQR) & $22.0(12.0-48.3)$ & $25.0(15.0-54.0)$ & $8.0(4.0-15.5)$ & $<0.001$ \\
\hline LolCU (day), median (IQR) & $5.5(3.0-14.0)$ & $5.0(3.0-14.0)$ & $6.0(4.0-14.0)$ & 0.36 \\
\hline Mechanical Ventilation, $n$ (\%) & $166(62)$ & $136(58)$ & $30(97)$ & $<0.001$ \\
\hline DoMV (day), median (IQR) & $3.0(0.0-9.0)$ & $2.0(0.0-9.0)$ & $6.0(3.0-14.0)$ & 0.001 \\
\hline Emergency operation, $n$ (\%) & $92(35)$ & $79(34)$ & $18(58)$ & 0.01 \\
\hline Angioembolization, $n$ (\%) & $57(21)$ & $48(20)$ & $9(29)$ & 0.35 \\
\hline $\mathrm{AKI}, n(\%)$ & $82(31)$ & $60(26)$ & $22(71)$ & $<0.001$ \\
\hline Hyperchloremia-48, n (\%) & $65(24)$ & $46(20)$ & $19(61)$ & $<0.001$ \\
\hline
\end{tabular}

$\mathrm{LoH}$ length of hospital stay, IQR interquartile range, LoICU length of intensive care unit stay, DoMV duration of mechanical ventilation, AKI acute kidney injury, Hyperchloremia-48 hyperchloremia 48 -h post-admission

Pro-inflammatory responses to hyperchloremic metabolic acidosis are mediated by nitric oxide and are associated with a higher interleukin (IL)-6 to IL-10 ratio when compared with lactic acidosis, as reported in in vitro cell models [15]. Hyperchloremic metabolic acidosis may be a pro-inflammatory modulator in sepsis $[6$, $16,17]$. Neutrophil function is also influenced by chloride influx through different chloride channels and via cotransporters [18, 19]. Low or absent extracellular chloride concentrations are associated with decreased neutrophil function [20,21].

Associations between fluid overload and morbidity and mortality were reported by the Fluid and Catheter Treatment Trial (FACTT) and other trials in surgical patients [22-24]. When administered in large volumes, NS has been shown to cause coagulopathy $[25,26]$. The mechanisms underlying saline-induced coagulopathy are not fully known. Our results showed that non-survivors had significantly higher total volumes of fluid infused compared to survivors $(7.4 \mathrm{~L}$ vs. $11.8 \mathrm{~L})$. However, these patients could have had greater fluid demands because of hypotension or the need to undergo emergency operations (which was significantly higher in non-survivors than in survivors). Shaw et al. [11] revealed that resuscitation volumes $\leq 1500 \mathrm{~mL}$, which may reflect a low total chloride load $(0-300 \mathrm{mmol})$, were not associated with increased mortality. However, there was a trend towards increasing mortality with increasing chloride load in patients receiving $\geq 1500 \mathrm{~mL}$ of fluid for resuscitation, which may reflect the potential risk of resuscitation with chloride-rich fluids. In the present study, the overall median total fluid infusion volume was 7.6 L (IQR 6.3-10.1 $\mathrm{L})$ over $48 \mathrm{~h}$. Total chloride loads may have been enough to show this trend, but we were unable to calculate the

Table 5 Univariate and multivariate analyses evaluating the association of hyperchloremia-48 on 30-day mortality

\begin{tabular}{|c|c|c|c|c|}
\hline \multirow[t]{2}{*}{ Variables } & \multicolumn{2}{|l|}{ Univariate analysis } & \multicolumn{2}{|l|}{ Multivariate analysis } \\
\hline & OR $(95 \% \mathrm{Cl})$ & $p$ value & OR $(95 \% \mathrm{Cl})$ & $p$ value \\
\hline Age (per year) & $1.014(0.994-1.034)$ & 0.17 & . & \\
\hline ISS & $1.080(1.033-1.130)$ & 0.001 & . & \\
\hline RTS & $0.621(0.511-0.754)$ & $<0.001$ & $0.715(0.551-0.929)$ & 0.01 \\
\hline Mechanical ventilation (yes) & $21.838(2.929-162.842)$ & 0.003 & . & \\
\hline Transfusion (yes) & $6.750(2.290-19.894)$ & 0.001 & . & \\
\hline Hypotension (SBP < 90, yes) & $4.477(1.971-10.168)$ & $<0.001$ & . & \\
\hline Hypothermia (BT<35 ${ }^{\circ} \mathrm{C}$, yes) & $3.828(1.626-9.012)$ & 0.002 & . & \\
\hline Cumulative fluid balance at $48 \mathrm{~h}$ (per $\mathrm{L}$ ) & $1.000(1.000-1.000)^{\mathrm{a}}$ & 0.01 & . & \\
\hline Base deficit at $48 \mathrm{~h}$ (per $1 \mathrm{mmol} / \mathrm{L})$ & $0.740(0.631-0.868)$ & $<0.001$ & $0.782(0.650-0.940)$ & 0.009 \\
\hline Hyperchloremia-48 (yes) & 8.667 (3.736-20.103) & $<0.001$ & $4.567(1.634-12.764)$ & 0.004 \\
\hline Use of vasopressor (yes) & $29.186(6.789-125.472)$ & $<0.001$ & $9.233(1.925-44.295)$ & 0.005 \\
\hline Use of loop diuretics (yes) & $3.243(1.432-7.344)$ & 0.005 & . & \\
\hline AKI (yes) & $7.130(3.112-16.336)$ & $<0.001$ & $2.574(0.650-0.940)$ & 0.08 \\
\hline
\end{tabular}

$O R$ odds ratio, $C I$ confidence interval, ISS injury severity score, $R T S$ revised trauma score, $A K I$ acute kidney injury, $S B P$ systolic blood pressure, $B T$ body temperature, $A K I$ acute kidney injury

a $(1.000027-1.000216)$

$p$ value for the Hosmer-Lemeshow goodness-of-fit-test was 0.256 
Table 6 Univariate and multivariate analyses evaluating the association of absolute value of Chloride (mmol/L) on 30-day mortality

\begin{tabular}{|c|c|c|c|c|}
\hline \multirow[t]{2}{*}{ Variables } & \multicolumn{2}{|l|}{ Univariate analysis } & \multicolumn{2}{|l|}{ Multivariate analysis } \\
\hline & OR $(95 \% \mathrm{Cl})$ & $p$ value & OR $(95 \% \mathrm{Cl})$ & $p$ value \\
\hline Age (per year) & $1.014(0.994-1.034)$ & 0.17 & . & \\
\hline ISS & $1.080(1.033-1.130)$ & 0.001 & & \\
\hline RTS & $0.621(0.511-0.754)$ & $<0.001$ & $0.720(0.552-0.939)$ & 0.02 \\
\hline Mechanical ventilation (yes) & $21.838(2.929-162.842)$ & 0.003 & . & \\
\hline Transfusion (yes) & $6.750(2.290-19.894)$ & 0.001 & $3.296(0.756-14.372)$ & 0.11 \\
\hline Hypotension (SBP < 90, yes) & $4.477(1.971-10.168)$ & $<0.001$ & & \\
\hline Hypothermia (BT < $35^{\circ} \mathrm{C}$, yes) & $3.828(1.626-9.012)$ & 0.002 & & \\
\hline Cumulative fluid balance at $48 \mathrm{~h}$ (per $1 \mathrm{~L}$ ) & $1.000(1.000-1.000)^{\mathrm{a}}$ & 0.01 & & \\
\hline Base deficit at $48 \mathrm{~h}$ (per $1 \mathrm{mmol} / \mathrm{L})$ & $0.740(0.631-0.868)$ & $<0.001$ & $0.748(0.619-0.905)$ & 0.003 \\
\hline Chloride at $48 \mathrm{~h}$ (per $1 \mathrm{mmol} / \mathrm{L})$ & $1.151(1.084-1.222)$ & $<0.001$ & $1.075(1.006-1.150)$ & 0.03 \\
\hline Use of vasopressor (yes) & $29.186(6.789-125.472)$ & $<0.001$ & $9.758(2.044-46.600)$ & 0.004 \\
\hline Use of loop diuretics (yes) & $3.243(1.432-7.344)$ & 0.005 & . & \\
\hline AKI (yes) & $7.130(3.112-16.336)$ & $<0.001$ & . & . \\
\hline
\end{tabular}

$O R$ odds ratio, $C l$ confidence interval, ISS injury severity score, $R T S$ revised trauma score, $A K I$ acute kidney injury, $S B P$ systolic blood pressure, $B T$ body temperature, AKI acute kidney injury

a (1.000027-1.000216)

$p$ value for the Hosmer-Lemeshow goodness-of-fit-test was 0.148

volume-adjusted chloride load. Some data were missing and we could not include other kinds of infused fluid, such as blood transfusion products and colloid.

Infusion of chloride-rich solutions, such as NS, is itself associated with hyperchloremia [1, 13, 27-29] which has been associated with poor patient outcomes $[8,9]$. Shaw et al. [30] considered postoperative outcomes following abdominal surgery: Their analysis used propensity score matching to match 926 patients receiving Plasma-Lyte in a 3:1 ratio to patients receiving NS. Patients receiving NS were more likely to suffer major postoperative complications, AKI and infection. Similarly, large changes in serum chloride levels correlated with greater in-hospital mortality [11]. However, a meta-analysis of high-versus low-chloride content in perioperative and critical care fluid resuscitation concluded that a weak but significant

Table 7 Univariate and multivariate analyses evaluating the association of $\Delta$ chloride $(\mathrm{mmol} / \mathrm{L})$ on 30 -day mortality

\begin{tabular}{|c|c|c|c|c|}
\hline \multirow[t]{2}{*}{ Variables } & \multicolumn{2}{|l|}{ Univariate analysis } & \multicolumn{2}{|l|}{ Multivariate analysis } \\
\hline & OR $(95 \% \mathrm{Cl})$ & $p$ value & OR $(95 \% \mathrm{Cl})$ & $p$ value \\
\hline Age (per year) & $1.014(0.994-1.034)$ & 0.17 & . & \\
\hline ISS & $1.080(1.033-1.130)$ & 0.001 & . & \\
\hline RTS & $0.621(0.511-0.754)$ & $<0.001$ & $0.728(0.559-0.950)$ & 0.02 \\
\hline Mechanical ventilation (yes) & $21.838(2.929-162.842)$ & 0.003 & . & \\
\hline Transfusion (yes) & $6.750(2.290-19.894)$ & 0.001 & . & \\
\hline Hypotension (SBP < 90mmHg, yes) & $4.477(1.971-10.168)$ & $<0.001$ & . & \\
\hline Hypothermia (BT<35 ${ }^{\circ} \mathrm{C}$, yes) & $3.828(1.626-9.012)$ & 0.002 & . & \\
\hline Cumulative fluid balance at $48 \mathrm{~h}$ (per $\mathrm{L}$ ) & $1.000(1.000-1.000)^{\mathrm{a}}$ & 0.01 & & \\
\hline Base deficit at $48 \mathrm{~h}$ (per $1 \mathrm{mmol} / \mathrm{L}$ ) & $0.740(0.631-0.868)$ & $<0.001$ & $0.761(0.631-0.918)$ & 0.004 \\
\hline$\Delta$ chloride (per $1 \mathrm{mmol} / \mathrm{L}$ ) & $1.165(1.096-1.238)$ & $<0.001$ & $1.096(1.027-1.169)$ & 0.006 \\
\hline Use of vasopressor (yes) & $29.186(6.789-125.472)$ & $<0.001$ & $10.392(2.222-48.604)$ & 0.003 \\
\hline Use of loop diuretics (yes) & $3.243(1.432-7.344)$ & 0.005 & & \\
\hline AKI (yes) & $7.130(3.112-16.336)$ & $<0.001$ & . & \\
\hline
\end{tabular}

$O R$ odds ratio, $C l$ confidence interval, ISS injury severity score, $R T S$ revised trauma score, $A K I$ acute kidney injury, SBP systolic blood pressure, $B T$ body temperature, $A K l$ acute kidney injury

a $(1.000027-1.000216)$

$p$ value for the Hosmer-Lemeshow goodness-of-fit-test was 0.835 
association of unfavorable outcomes was found, but that mortality was unaffected by chloride content [31]. Our results showed significant differences between survivors and non-survivors with the groups of $\Delta$ chloride. These findings contribute to the evidence of the potentially serious clinical impact of using chloride-rich crystalloids that could induce hyperchloremia.

The present study has a number of limitations. First, this was a retrospective, single tertiary center study. Second, the fluid resuscitation strategy could not be controlled because of retrospective data collection. Similarly, prehospital fluid resuscitation was not included. However, we did exclude patients with hyperchloremia at initial measurement on admission. Third, as mentioned previously, we were unable to include other kinds of infused fluid, such as blood products and colloids. We were also unable to calculate the volume-adjusted chloride load. Fourth, selection bias may have been possible given the inclusion and exclusion criteria. Lastly, because of the limited patient numbers, our results are not representative of all major trauma patients. Larger, controlled studies are need for greater power and reliability.

\section{Conclusion}

Hyperchloremia 48-h post-admission occurs frequently in patients who have sustained major trauma. We found that hyperchloremia- 48 and $\Delta$ chloride were independently associated with all cause 30-day mortality in major trauma patients. These variables might be useful to predict mortality in patients who have sustained major trauma. However, data from large, prospective, controlled studies that are adequately powered are needed to detect differences in outcomes such as mortality and morbidity.

\section{Abbreviations}

AKI: Acute kidney injury; APACHE II: Acute Physiology and Chronic Health Evaluation II; Cl: Confidence interval; GCS: Glasgow coma scale; ICU: Intensive care unit; IL: Interleukin; IQR: Inter-quartile range; ISS: Injury severity score; NS: Normal saline; OR: Odds ratio; RTS: Revised trauma score; TRISS: Trauma and injury severity score

\section{Acknowledgements}

Not applicable.

\section{Funding}

Not applicable.

\section{Availability of data and material}

The datasets analyzed in the current study are available on reasonable request to the corresponding author.

\section{Authors' contributions}

JYL and SHL designed the study, JYL, THH, KYL collected the data. JYL did the statistical analyses with SHL, JGL and MJJ. JYL wrote the article. All authors read and approved the submitted version.

\section{Competing interests}

The authors declare that they have no competing interests.

\section{Ethics approval and consent to participate}

The study was approved by the Institutional Review Board (IRB No. 4-2016-0295), which waived the requirement for informed consent because of the retrospective nature of the study.

Received: 18 July 2016 Accepted: 28 September 2016

Published online: 04 October 2016

\section{References}

1. Yunos NM, Bellomo R, Story D, Kellum J. Bench-to-bedside review: Chloride in critical illness. Crit Care. 2010;14:226.

2. Berend K, van Hulsteijn LH, Gans RO. Chloride: the queen of electrolytes? Eur J Intern Med. 2012;23:203-11.

3. Wilkes NJ, Woolf RL, Powanda MC, Gan TJ, Machin SJ, Webb A, Mutch M, Bennett-Guerrero E, Mythen M. Hydroxyethyl starch in balanced electrolyte solution (Hextend)-pharmacokinetic and pharmacodynamic profiles in healthy volunteers. Anesth Analg. 2002;94:538-44. Table of contents.

4. Wilcox CS. Regulation of renal blood flow by plasma chloride. J Clin Invest. 1983;71:726-35.

5. Bullivant EM, Wilcox CS, Welch WJ. Intrarenal vasoconstriction during hyperchloremia: role of thromboxane. Am J Physiol. 1989;256:F152-7.

6. Kellum JA, Song M, Almasri E. Hyperchloremic acidosis increases circulating inflammatory molecules in experimental sepsis. Chest. 2006;130:962-7.

7. Boniatti MM, Cardoso PR, Castilho RK, Vieira SR. Is hyperchloremia associated with mortality in critically ill patients? A prospective cohort study. J Crit Care. 2011:26:175-9.

8. Neyra JA, Canepa-Escaro F, Li X, Manllo J, Adams-Huet B, Yee J, Yessayan L, Acute Kidney Injury in Critical IIIness Study G. Association of Hyperchloremia with hospital mortality in critically ill septic patients. Crit Care Med. 2015;43: 1938-44.

9. McCluskey SA, Karkouti K, Wijeysundera D, Minkovich L, Tait G, Beattie WS. Hyperchloremia after noncardiac surgery is independently associated with increased morbidity and mortality: a propensity-matched cohort study. Anesth Analg. 2013;117:412-21.

10. Subcommittee A, American College of Surgeons' Committee on T, International Awg. Advanced trauma life support (ATLS(R)): the ninth edition. J Trauma Acute Care Surg. 2013;74:1363-6.

11. Shaw AD, Raghunathan K, Peyerl FW, Munson SH, Paluszkiewicz SM, Schermer CR. Association between intravenous chloride load during resuscitation and in-hospital mortality among patients with SIRS. Intensive Care Med. 2014; 40:1897-905.

12. Young JB, Utter GH, Schermer CR, Galante JM, Phan HH, Yang Y, Anderson $B A$, Scherer LA. Saline versus Plasma-Lyte A in initial resuscitation of trauma patients: a randomized trial. Ann Surg. 2014;259:255-62.

13. Scheingraber $\mathrm{S}$, Rehm M, Sehmisch C, Finsterer U. Rapid saline infusion produces hyperchloremic acidosis in patients undergoing gynecologic surgery. Anesthesiology. 1999:90:1265-70.

14. Bellomo R, Ronco C, Kellum JA, Mehta RL, Palevsky P. Acute renal failure definition, outcome measures, animal models, fluid therapy and information technology needs: the Second International Consensus Conference of the Acute Dialysis Quality Initiative (ADQI) Group. Crit Care. 2004:8:R204-12.

15. Kellum JA, Song M, Li J. Lactic and hydrochloric acids induce different patterns of inflammatory response in LPS-stimulated RAW 264.7 cells. Am J Physiol Regul Integr Comp Physiol. 2004;286:R686-92.

16. Pedoto A, Nandi J, Oler A, Camporesi EM, Hakim TS, Levine RA. Role of nitric oxide in acidosis-induced intestinal injury in anesthetized rats. J Lab Clin Med. 2001;138:270-6.

17. Pedoto A, Caruso JE, Nandi J, Oler A, Hoffmann SP, Tassiopoulos AK, McGraw DJ, Camporesi EM, Hakim TS. Acidosis stimulates nitric oxide production and lung damage in rats. Am J Respir Crit Care Med. 1999;159:397-402

18. Aiken ML, Painter RG, Zhou Y, Wang G. Chloride transport in functionally active phagosomes isolated from Human neutrophils. Free Radic Biol Med. 2012;53:2308-17.

19. Sun YT, Shieh CC, Delpire E, Shen MR. K(+)-Cl(-) cotransport mediates the bactericidal activity of neutrophils by regulating NADPH oxidase activation. J Physiol. 2012;590:3231-43.

20. Painter RG, Bonvillain RW, Valentine VG, Lombard GA, LaPlace SG, Nauseef WM, Wang G. The role of chloride anion and CFTR in killing of Pseudomonas aeruginosa by normal and CF neutrophils. J Leukoc Biol. 2008;83:1345-53. 
21. Akong-Moore K, Chow OA, von Kockritz-Blickwede M, Nizet V. Influences of chloride and hypochlorite on neutrophil extracellular trap formation. PLoS One. 2012;7:e42984.

22. Brandstrup B, Tonnesen $H$, Beier-Holgersen $R$, Hjortso E, Ording H, LindorffLarsen K, Rasmussen MS, Lanng C, Wallin L, Iversen LH, et al. Effects of intravenous fluid restriction on postoperative complications: comparison of two perioperative fluid regimens: a randomized assessor-blinded multicenter trial. Ann Surg. 2003;238:641-8.

23. National Heart L, Blood Institute Acute Respiratory Distress Syndrome Clinical Trials N, Wiedemann HP, Wheeler AP, Bernard GR, Thompson BT, Hayden D, de Boisblanc B, Connors Jr AF, Hite RD, Harabin AL. Comparison of two fluidmanagement strategies in acute lung injury. N Engl J Med. 2006;354:2564-75.

24. Stewart RM, Park PK, Hunt JP, McIntyre Jr RC, McCarthy J, Zarzabal LA Michalek JE, National Institutes of Health/National Heart L, Blood Institute Acute Respiratory Distress Syndrome Clinical Trials N. Less is more: improved outcomes in surgical patients with conservative fluid administration and central venous catheter monitoring. J Am Coll Surg. 2009;208:725-35. discussion 735-727.

25. Ahn HJ, Yang M, Gwak MS, Koo MS, Bang SR, Kim GS, Lee SK. Coagulation and biochemical effects of balanced salt-based high molecular weight vs saline-based low molecular weight hydroxyethyl starch solutions during the anhepatic period of liver transplantation. Anaesthesia. 2008; 63:235-42.

26. Todd SR, Malinoski D, Muller PJ, Schreiber MA. Lactated Ringer's is superior to normal saline in the resuscitation of uncontrolled hemorrhagic shock. J Trauma. 2007:62:636-9.

27. Williams EL, Hildebrand KL, McCormick SA, Bedel MJ. The effect of intravenous lactated Ringer's solution versus $0.9 \%$ sodium chloride solution on serum osmolality in human volunteers. Anesth Analg. 1999;88:999-1003.

28. Reid F, Lobo DN, Williams RN, Rowlands BJ, Allison SP. (Ab)normal saline and physiological Hartmann's solution: a randomized double-blind crossover study. Clin Sci (Lond). 2003;104:17-24.

29. Chowdhury AH, Cox EF, Francis ST, Lobo DN. A randomized, controlled, double-blind crossover study on the effects of 2-L infusions of $0.9 \%$ saline and plasma-lyte(R) 148 on renal blood flow velocity and renal cortical tissue perfusion in healthy volunteers. Ann Surg. 2012;256:18-24.

30. Shaw AD, Bagshaw SM, Goldstein SL, Scherer LA, Duan M, Schermer CR Kellum JA. Major complications, mortality, and resource utilization after open abdominal surgery: $0.9 \%$ saline compared to Plasma-Lyte. Ann Surg. 2012;255:821-9

31. Krajewski ML, Raghunathan K, Paluszkiewicz SM, Schermer CR, Shaw AD. Meta-analysis of high- versus low-chloride content in perioperative and critical care fluid resuscitation. Br J Surg. 2015;102:24-36.

\section{Submit your next manuscript to BioMed Central and we will help you at every step:}

- We accept pre-submission inquiries

- Our selector tool helps you to find the most relevant journal

- We provide round the clock customer support

- Convenient online submission

- Thorough peer review

- Inclusion in PubMed and all major indexing services

- Maximum visibility for your research

Submit your manuscript at www.biomedcentral.com/submit

C Biomed Central 